\title{
Case Report: A Long Survivor Patient with Stage IV Gastric Adenocarcinoma
}

\author{
Marta Pina, Maria Leitão, Manuela Machado \\ Medical Oncology Department, Portuguese Institute of Oncology of Porto, Porto, Portugal \\ Email: m.machado.fn@gmail.com
}

How to cite this paper: Pina, M., Leitão, M. and Machado, M. (2020) Case Report: A Long Survivor Patient with Stage IV Gastric Adenocarcinoma. Journal of Cancer Therapy, 11, 357-364.

https://doi.org/10.4236/jct.2020.116030

Received: May 6, 2020

Accepted: May 26, 2020

Published: May 29, 2020

Copyright (c) 2020 by author(s) and Scientific Research Publishing Inc. This work is licensed under the Creative Commons Attribution International License (CC BY 4.0).

http://creativecommons.org/licenses/by/4.0/

(c) (i) Open Access

\begin{abstract}
Gastric cancer (GC) is the fifth most common malignancy of the world and third leading cause of cancer death. At diagnosis, 35\% of GC patients have distant metastases and in these cases the survival rate is very poor with a median overall survival (OS) inferior to 1 year. We report a case of a 67-year-old woman with gastric carcinoma initially deemed limited stage on diagnosis (cT2N0M0), treated surgically with radical subtotal gastrectomy with Billroth II reconstruction. In the staging CT scan, the patient presented a liver image that was considered benign. Three months later, due to abdominal pain, the patient performed another $\mathrm{CT}$ scan and the diagnostic of a large single liver metastasis was made; retrospectively it was observed that the lesion was present at diagnosis and that it had increased. A biopsy was performed which confirmed the metastatic origin. In a multidisciplinary team, the lesion was considered unresectable. She was proposed for first line $\left(1^{\text {st }} \mathrm{L}\right)$ palliative chemotherapy (ChT) with FOLFIRI, with partial response as best response. After 30 cycles of FOLFIRI, bone metastases were diagnosed. The patient was submitted to a cementoplasty of D11-12 and L1-2. Afterwards, she started $2^{\text {nd }} \mathrm{L}$ ChT with mFOLFOX6 and at the same time she started zoledronic acid every 28 days. The best response to mFOLFOX6 was stable disease. Since November 2018, the patient has been treated with zoledronic acid every 28 days alone and maintains stable disease without ChT.
\end{abstract}

\section{Keywords}

Gastric, Cancer, Metastatic Gastric Cancer, Palliative Surgery, Palliative Chemotherapy, Survival

\section{Introduction}

Gastric cancer is the fifth most common malignancy in the world and the third 
leading cause of cancer death, having a high fatality-to-case ratio (70\%). At the time of diagnosis, $35 \%$ of gastric cancer patients have evidence of distant metastases, with about $14 \%$ of the synchronous metastases confined to the liver. Metastatic gastric cancer has an abysmal 5-year survival rate that rarely exceeds 5\% and the median overall survival (OS) is less than 1 year. Little progress has been made in the treatment of advanced or metastatic cancer and prognosis remains poor. Current management options include palliative surgery, systemic chemotherapy (ChT), investigational therapies or best supportive care (BSC). Several studies show that in metastatic setting, the 5-year survival is longer in the group of patients submitted to metastasectomy and palliative gastrectomy compared to gastrectomy alone [1]-[8].

In metastatic disease, $\mathrm{ChT}$ is associated with an increase in OS and quality of life (QoL) when compared to BSC. There is no consensus regarding the best ChT scheme in the 1st line. Poly-chemotherapy schemes are associated with an increase in OS, but usually also with more toxicity. The most commonly used are platinum and fluoropyrimidine based doublets. In adenocarcinoma with HER 2 overexpression trastuzumab should be considered. Schemes with irinotecan or taxanes are also options and in patients with performance status (PS) 0 triple therapy may be considered [9].

In this article, we report a long-term surviving case of metastatic gastric cancer. This will allow a reflection about the fundamental nature of individualizing treatment and the importance of a multidisciplinary approach of a patient that was treated with a radical intuit but was discovered later to have been palliative ad initium.

\section{Case Report}

In December 2016, a woman without relevant pathological backgrounds, born in 1952, was diagnosed with gastric adenocarcinoma in an upper endoscopy made because she presented epigastric pain. In the thoraco-abdomino-pelvic CT scan of staging performed, the patient presented a liver image that was assumed as benign (Figure 1). In a multidisciplinary team, the patient was proposed to surgery

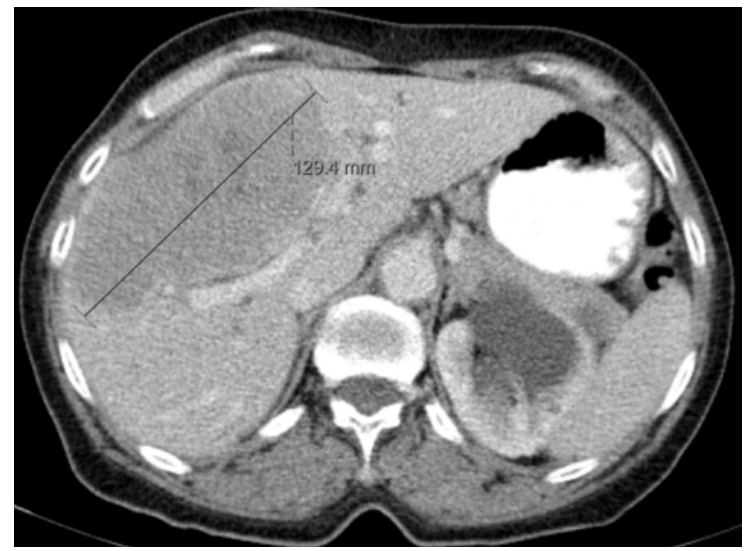

Figure 1. Hepatic lesion assumed as benign (hemangioma) on the staging CT scan. 
without staging laparoscopy because it was assumed as limited stage (cT2N0M0).

She was submitted to an exploratory laparotomy that identified a malignant lesion of incisura angularis with extension to the posterior gastric wall. The surgeons identified the hepatic lesion in the right lobe, but it did not present suspicious characteristics, which was in agreement with the radiological description of a large hemangioma. She underwent a radical subtotal gastrectomy with Billroth II reconstruction. The pathologic examination revealed a gastric adenocarcinoma, grade 2, no metastasized lymph nodes on the 47 excised, pT3N0cM0, stage IIA, according to AJCC staging classification $7^{\text {th }}$ edition [10]. The patient was kept in follow up, without adjuvant treatments.

Three months later, the patient presented intense abdominal pain located to the upper quadrants. The patient was very symptomatic (abdominal pain) and hepatomegaly was evident on physical examination. A thoraco-abdominal-pelvic CT scan and an 18F-Fluorodeoxyglucose Positron Emission Tomography (PET-CT) were requested.

On the CT scan, a large single liver metastasis was identified; retrospectively it was observed that the lesion was present at the diagnosis and that it had increased (Figure 2). On PET-CT, a mass occupying most of the right hepatic lobe with high FDG hypermetabolism was diagnosed (Figure 3). A liver biopsy was performed which confirmed the metastatic origin.

In a multidisciplinary team discussion, the liver metastases were considered unresectable, and the patient was proposed to palliative ChT. The patient was very symptomatic with abdominal pain and a Performance Status (PS) of 2. She started prednisolone $20 \mathrm{mg}$ twice daily (bid), omeprazole $40 \mathrm{mg}$ once daily (id), morphine sulfate $60 \mathrm{mg}$ bid. After discussing potential benefits and risks, it was decided to start palliative ChT. The expression of HER 2 was not yet available and the patient was afraid of neuropathy, so the scheme chosen was FOLFIRI (irinotecan/calcium folinate (LV)/5 fluorouracil (5-FU)), which she started in June 2017. The ChT was very well tolerated and the best response was partial response on CT scan (Figure 4), accompanied by a great improvement in quality

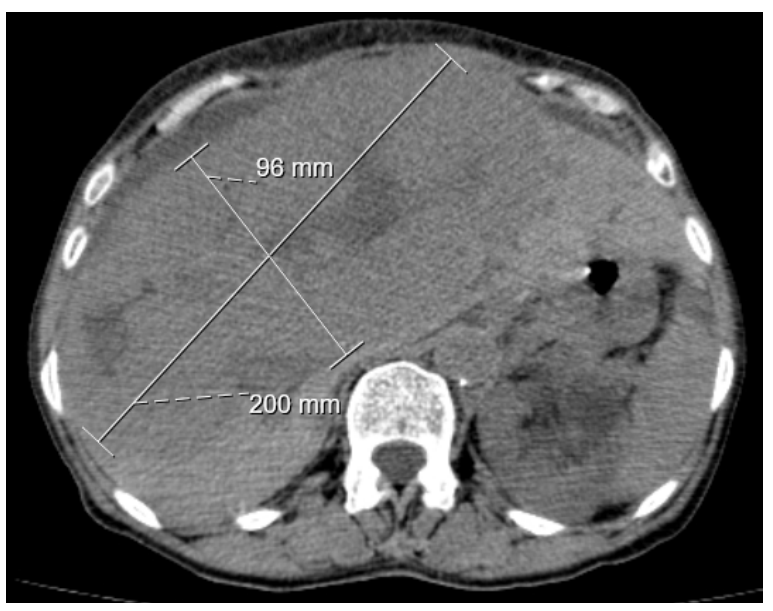

Figure 2. Abdominal CT image showing the growth of the liver mass. 


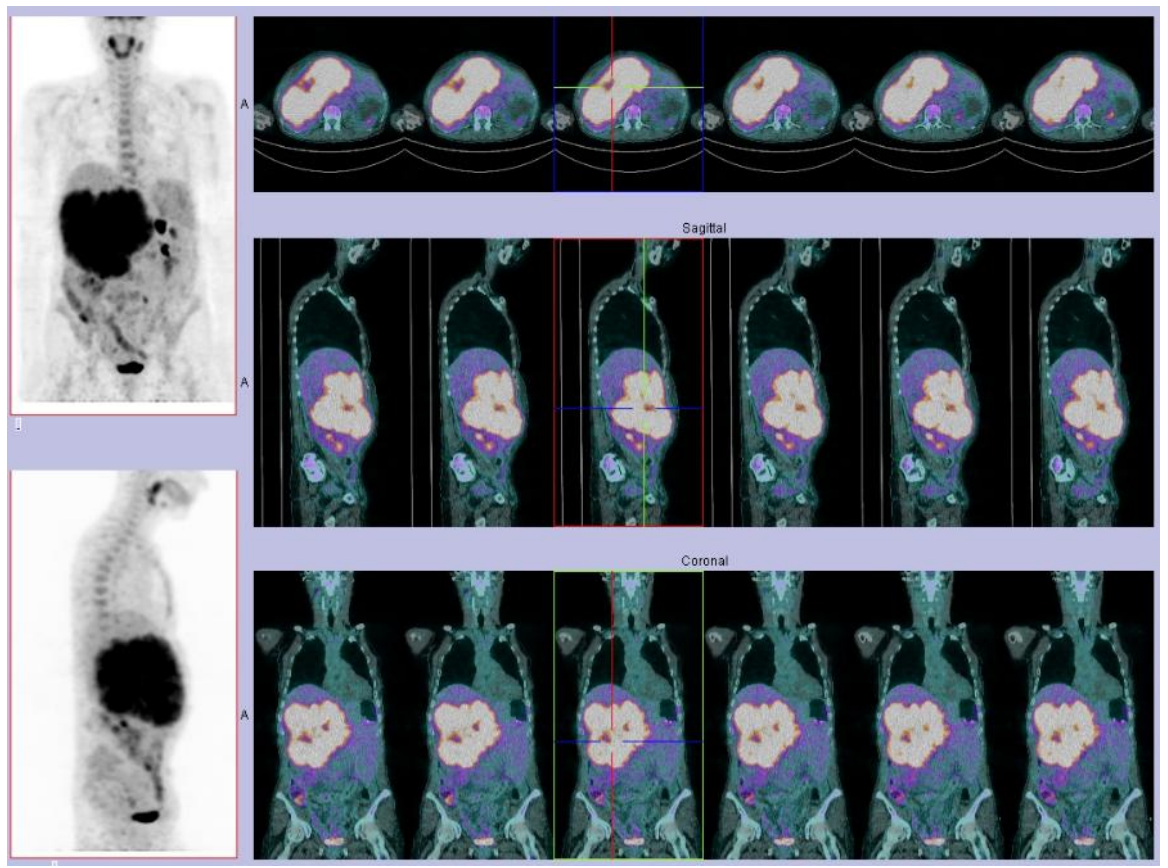

Figure 3. PET-CT showing a mass occupying most of the right hepatic lobe with high FDG hypermetabolism.

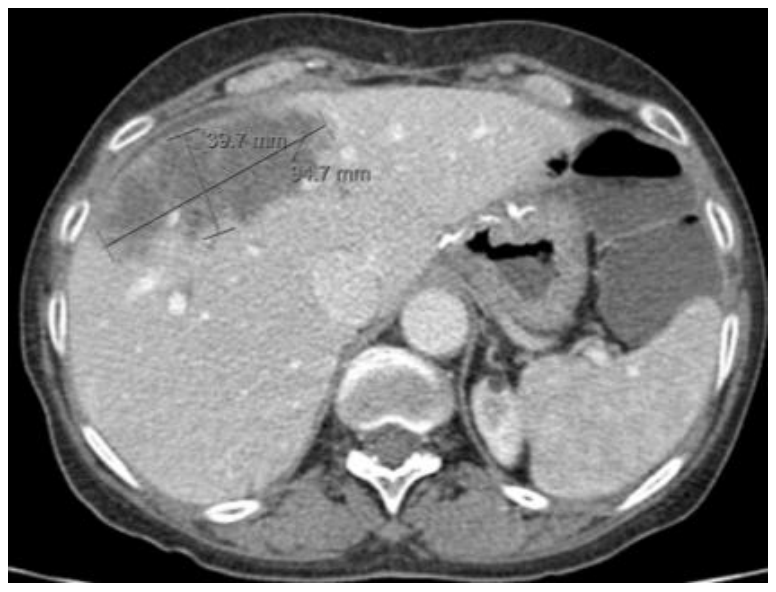

Figure 4. Evolution of hepatic metastasis after 5 cycles of $1^{\text {st }} \mathrm{L}$ palliative ChT.

of life (QoL) and in pain control. In October 2018 the patient had PS 0 and the only medications needed were omeprazole $20 \mathrm{mg}$ id and prednisolone $5 \mathrm{mg}$ id. FOLFIRI schedule was maintained until November 2018 (30 cycles). In October 2018, the patient developed dorsal and lumbar pain. She underwent a thoraco-abdominal-pelvic and spinal CT, where she presented multiple new vertebral bone lesions, with stability of the hepatic mass and no evidence of other new lesions; disease progression was assumed.

In the multidisciplinary team discussion, she was proposed to surgery. On the $21^{\text {st }}$ of February 2018, she was submitted to a cementoplasty of D11-12 and L1-2. Even though it was requested, no lesion biopsy was performed.

In March 2018, she started $2^{\text {nd }} \mathrm{L}$ ChT with mFOLFOX6 (oxaliplatin/LV/5 FU) 
and zoledronic acid every 28 days. The best response to mFOLFOX6 was partial response of the hepatic lesion (Figure 5) and stable bone disease (on CT scan and on bone scintigraphy). This schedule of ChT was also very well tolerated and the patient completed 12 cycles. Since November 2018, the patient is only being treated with zoledronic acid every 28 days and maintains stable disease on CT scan. At this time, April 2020, she has a PS 1, she is taking omeprazole $20 \mathrm{mg}$ id, prednisolone $10 \mathrm{mg}$ id and tapentadol $200 \mathrm{mg}$ bid.

\section{Discussion}

Historically, the role of surgery in patients with metastatic cancer was predominantly limited to palliative or emergent interventions and different international guidelines support different approaches regarding surgical resection in these patients. Resection of the primary tumor in patients with metastatic disease combined with newer and more efficient chemotherapy regimens may confer survival benefit and increased QoL. However, the surgical treatment must be offered only to well-selected patients, who might benefit the most, considering tumor and patient characteristics, due to potential surgical risks and morbility. A study has found that palliative gastrectomy for tumors confined to the serosa (T1-3) had a favorable effect on median survival (18.9 versus 10.2 months, $\mathrm{P}<0.001$ ). However, in T4 disease (invasion beyond serosa), gastrectomy had no improvement in survival compared to palliative chemotherapy alone (11.8 versus 8.8 months, $\mathrm{P}=0.351)$. T4 stage is a negative prognostic factor and should be considered as a contraindication for surgery in metastatic gastric cancer (MGC) to the liver due to high risk of concurrent peritoneal disease [7] [8] [11].

The National Comprehensive Cancer Network (NCCN) guidelines recommend that gastric resections should be reserved for resectable disease. On the other hand, Japanese Gastric Cancer Association (JGCA) guidelines suggest that surgery should be considered in certain patients in whom it may be beneficial; the issue remaining the selection of patients [10].

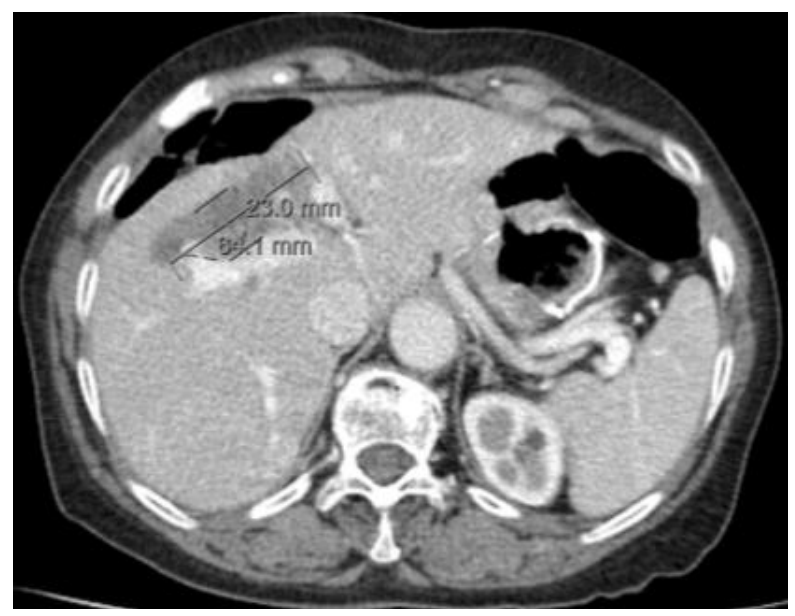

Figure 5. Evolution of hepatic metastasis after 3 months after discontinuation of $2^{\text {nd }} L$ ChT. 
As previously mentioned, in MGC, $\mathrm{ChT}$ is associated with an increase in OS and QoL when compared to BSC. There is no consensus regarding the best ChT scheme in $1^{\text {st }} \mathrm{L}$. Poly-chemotherapy schemes are associated with an increase in OS but usually also with more toxicity. The most commonly used are platinum and fluoropyrimidine based doublets, but the regimen should be chosen and adapted taking into account the patient's characteristics and preferences [12] [13] [14] [15].

Zoledronic acid may be considered in patients with bone metastasis. Biphosphonates may improve QoL and pain control, as well as decrease the frequency of skeletal events such as fractures [16] [17].

The present clinical case is a peculiar one, because statistically most patients die within a year after diagnosis; however, this patient is alive and with a good PS three and half years after diagnosis [1] [15]. Little is known about the characteristics of the patients who survive beyond 3 years. There are some studies that try to review these data, but most of the samples are small and homogeneous (primarily Asian populations), limiting the interpretation. These published studies suggest several independent prognostic factors: age, performance status, initial stage, surgery, tumor size, lymph node involvement, non-diffuse histology, presence of metastases and good response do ChT, may be related to better outcomes [18] [19].

In this particular case, the outcome can be justified by a multidisciplinary approach to a patient with $<65$ years, an initial PS 2 (which was a consequence of the oncologic disease, showing improvement with treatment), a T3 tumor submitted to a radical surgery resulting in good local control, no lymph node involvement and a favorable histology (non-diffuse). Despite the liver and (later) bone metastasis, the disease responded well to $\mathrm{ChT}$ with a sustained partial response.

The reported case invites reflection on the different evolutions of apparently similar tumors, using limited histological and molecular classifications. Perhaps in the future, genomic, epigenomic and transcriptomic information will provide us with the tools to recognize the intrinsic differences in seemingly analogous cases. Until then, we must rely on imperfect classifications and clinical expertise to allocate the best decisions to each case. Surgical and medical interventions should be carefully discussed in multidisciplinary teams, in order to achieve personalized care for the patients. This is of particular importance in patients with advanced disease, in which every choice must be well thought out to provide the maximum QoL and survival, while minimizing toxicity and morbility.

This report communicates an evident case of personalized medicine, of adaptation of canon and integration of guidelines with real life events, achieving a very long survival with good QoL in a patient with an advanced disease ad initium.

\section{Conclusions}

Gastric metastatic cancer usually carries poor prognosis and radical surgery 
should be considered whenever possible to achieve a complete removal of the disease. A meta-analysis showed that palliative gastrectomy had a statistically significant survival benefit in patients with incurable advanced gastric carcinoma, especially in metastatic patients. Survival advantage is longer when chemotherapy was used. For patients with liver metastasis, palliative gastrectomy may provide better results regarding survival compared with patients with metastasis in other organs.

When surgery is not possible or not considered, palliative chemotherapy offers a good benefit in prolonging survival and quality of life. This case is a good example of disease control and sustained response. Clinical cases like this are more and more frequent and reflect the need for personalized medicine.

At the time when precision medicine is being discussed according to the molecular profile of the tumor, the authors considered that the clinical profile of each patient is also fundamental in the decision making process.

\section{Conflicts of Interest}

The authors declare no conflicts of interest regarding the publication of this paper.

\section{References}

[1] Siegel, R.L., Miller, K.D. and Jemal, A. (2017) Cancer Statistics, 2017. CA: A Cancer Journal for Clinicians, 67, 7-30. https://doi.org/10.3322/caac.21387

[2] Ferlay, J., et al. (2015) Cancer Incidence and Mortality Worldwide: Sources, Methods and Major Patterns in GLOBOCAN 2012. International Journal of Cancer, 136, E359-E386. https://doi.org/10.1002/ijc.29210

[3] Guggenheim, D.E. and Shah, M.A. (2013) Gastric Cancer Epidemiology and Risk Factors. Journal of Surgical Oncology, 107, 230-236. https://doi.org/10.1002/jso.23262

[4] Torre, L.A., et al. (2015) Global Cancer Statistics, 2012. CA: A Cancer Journal for Clinicians, 65, 87-108. https://doi.org/10.3322/caac.21262

[5] Arnold, M., et al. (2015) Recent Trends in Incidence of Five Common Cancers in 26 European Countries since 1988: Analysis of the European Cancer Observatory. European Journal of Cancer, 51, 1164-1187. https://doi.org/10.1016/j.ejca.2013.09.002

[6] D’Angelica, M., et al. (2004) Patterns of Initial Recurrence in Completely Resected Gastric Adenocarcinoma. Annals of Surgery, 240, 808-816.

https://doi.org/10.1097/01.sla.0000143245.28656.15

[7] Picado Roque, O., et al. (2018) The Role of Surgical Resection for Stage IV Gastric Cancer with Synchronous Hepatic Metastasis. Journal of Surgical Research, 232, 422-429. https://doi.org/10.1016/j.jss.2018.06.067

[8] Gadde, R., et al. (2015) Metastatic Gastric Cancer (MGC) Patients: Can We Improve Survival by Metastasectomy? A Systematic Review and Meta-Analysis. Jour nal of Surgical Oncology, 112, 38-45. https://doi.org/10.1002/jso.23945

[9] Machado, M. and Macedo, J. (2019) Treatment of Gastroesophageal Junction Tumors: A Commentary. British Journal of Cancer Research, 2, 244-245.

https://doi.org/10.31488/bjcr.124 
[10] Edge, S.B. and Compton, C.C. (2010) The American Joint Committee on Cancer: The 7th Edition of the AJCC Cancer Staging Manual and the Future of TNM. Annals of Surgical Oncology, 17, 1471-1474. https://doi.org/10.1245/s10434-010-0985-4

[11] Sun, J., et al. (2013) Clinical Significance of Palliative Gastrectomy on the Survival of Patients with Incurable Advanced Gastric Cancer: A Systematic Review and Meta-Analysis. BMC Cancer, 13, Article No. 577. https://doi.org/10.1186/1471-2407-13-577

[12] Beretta, E., et al. (2006) Irinotecan, Fluorouracil and Folinic Acid (FOLFIRI) as Effective Treatment Combination for Patients with Advanced Gastric Cancer in Poor Clinical Condition. Tumori, 92, 379-383. https://doi.org/10.1177/030089160609200502

[13] Yan, D. and Dai, H. (2009) FOLFOX Regimen in the Patients with Locally Advanced or Metastatic Gastric Cancer. Chinese Journal of Oncology, 31, 217-219.

[14] Kanagavel, D., et al. (2015) Second-Line Treatment of Metastatic Gastric Cancer: Current Options and Future Directions. World Journal of Gastroenterology, 21, 11621-11635. https://doi.org/10.3748/wjg.v21.i41.11621

[15] DeVita, V.T., Lawrence, T.S. and Rosenberg, S.A. (2015) Devita, Hellman, and Rosenberg's Cancer: Principles \& Practice of Oncology. Wolters Kluwer, Philadelphia, 1386-1446.

[16] Silvestris, N., et al. (2013) Natural History of Malignant Bone Disease in Gastric Cancer: Final Results of a Multicenter Bone Metastasis Survey. PLoS ONE, 8, e74402. https://doi.org/10.1371/journal.pone.0074402

[17] Polascik, T.J. and Mouraviev, V. (2008) Zoledronic Acid in the Management of Metastatic Bone Disease. Therapeutics and Clinical Risk Management, 4, 261-268. https://doi.org/10.2147/TCRM.S2707

[18] Kunz, P.L., et al. (2012) Long-Term Survivors of Gastric Cancer: A California Population-Based Study. Journal of Clinical Oncology, 30, 3507-3515. https://doi.org/10.1200/JCO.2011.35.8028

[19] Ina, K., et al. (2019) Long-Term Survivors of Metastatic Gastric Cancer for $>5$ Years after Chemotherapy Initiation. Cancer Reports and Reviews, 3, 1-5. https://doi.org/10.15761/CRR.1000183 